\title{
Kasuistiken
}

Ophthalmologe $2021 \cdot 118: 593-596$ https://doi.org/10.1007/s00347-020-01165-8 Online publiziert: 23. Juli 2020

(c) Der/die Autor(en) 2020

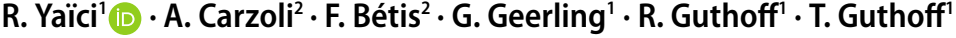

' Klinik für Augenheilkunde, Universitätsklinikum Düsseldorf, Düsseldorf, Deutschland

${ }^{2}$ service d'ophtalmologie, CHPG, Monaco, Monaco

\section{Atypische Neuritis nervi optici (NNO): Bedeutung einer umfassenden Abklärung}

\section{Falldarstellung}

\section{Anamnese}

Eine 65-jährige Frau wurde von ihrem niedergelassenen Augenarzt wegen einer bilateralen Papillenschwellung und plötzlicher beidseitiger Sehminderung innerhalb von wenigen Stunden überwiesen. Zusätzlich klagte die Patientin über Übelkeit und Erbrechen. Kopfschmerzen wurden von der Patientin verneint. Die Anamnese ergab außer einem moderaten Nikotinabusus keine Auffälligkeiten. Einige Tage zuvor hatte die Patientin eine Impfung mit Revaxis ${ }^{\circledR}$, Sanofi (Paris, Frankreich), gegen Diphtherie, Tetanus und Poliomyelitis erhalten.

\section{Befund}

Der Visus war am rechten Auge auf 0,1, am linken auf Fingerzählen reduziert. Der Ishihara-Test zeigte eine bilaterale Rot-Grün-Schwäche und einen relativen afferenten Pupillendefekt links. Am Fundus zeigte sich beidseits eine ausgeprägte Papillenschwellung mit peripapillären flammenförmigen Hämorrhagien ohne Zeichen einer Vaskulitis, Vitritis oder Netzhaut-/Aderhautbeteiligung. In der Fluoreszeinangiographie (FAG) stellten sich Papillenleckagen (• Abb. 1) dar. Eine kranielle und spinale MRT ergab den Verdacht auf eine bilaterale Sehnervenentzündung ohne Hinweis auf eine transverse Myelitis, zerebrale Beteiligung oder Raumforderung. Eine Biopsie der A. temporalis superficialis, eine Lumbalpunktion mit Liquordruckmessung und eine kardiovaskuläre Abklärung waren unauffällig. Labordiagnostisch zeigten sich keine erhöhten Entzündungs- oder Infektionsparameter (Borrelien, Lues, Bartonellen, Toxoplasmose) Auch AntiNeutrophile cytoplasmatische Antikörper (ANCA), antinukleärer Antikörper (ANA), nativer Anti-DNA-Ab und extrahierbare nukleäre Antigene(ENA) waren negativ. Die Bestimmung von Aquaporin 4(AQP4)- und Myelin-Oligodendrozyten-Glykoprotein(MOG)Antikörpern ergab schließlich den Nachweis von MOG-IgG.

\section{Diagnose}

Wir stellten die Diagnose einer AntiMOG-Antikörper-vermittelten Neuritis nervi optici, einer Neuromyelitis-opticaSpektrum-Erkrankung (NMOSD).
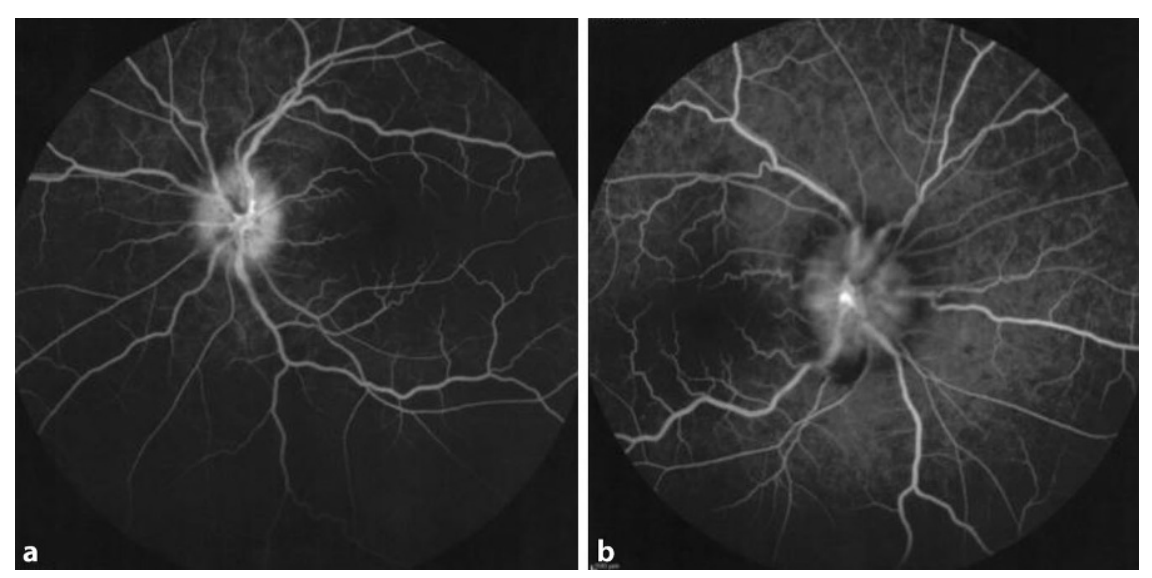

Abb. 1 ॥ Isoliertes Papillenödem in der Frühphase der Fluoreszeinangiographie (3 Tage nach Einsetzen der Kortisonstoßtherapie)

\section{Therapie und Verlauf}

Eine hoch dosierte intravenöse Methylprednisolon-Therapie $1 \mathrm{~g} /$ Tag für 5 Tage und anschließend $1 \mathrm{mg} / \mathrm{kgKG}$ über 4 Wochen ausschleichend reduziert, führte zu einer Visuserholung auf rechts 0,8 und links 1,0 und einem Rückgang der Papillenschwellung innerhalb von 4 Wochen. Die $30^{\circ}$-Perimetrie war 4 Wochen nach Therapie rechts nahezu regelrecht, links bestand noch ein bogenförmiges Skotom unten mit einem vergrößerten blinden Fleck (• Abb. 2).

\section{Diskussion}

In unserem Fall handelte es sich nach den durch das International Panel for NMO Diagnosis (IPND) neu definierten Kriterien von 2015 [1] um eine Neuromyelitis-optica-Spektrum-Erkrankung (engl. „neuromyelitis optica spectrum disorders" [NMOSD]). Bis 2006 wurde diese 
Erkrankung auch als Neuromyelitis optica (NMO) oder historisch als DevicKrankheit bezeichnet [2].

Kardinalsymptom dieses Krankheitskomplexes auf ophthalmologischem Fachgebiet ist die atypische Neuritis nervi optici $[2,3]$.

Die typische Neuritis nervi optici, wie sie bei der multiplen Sklerose (MS) vorkommt, zeigt eine einseitige Visusminderung mit Bulbusbewegungsschmerz, relativem afferentem pupillärem Defizit, Farbentsättigung und Zentralskotom bei Frauen in jungem Alter [4]. Von einer atypischen Neuritis nervi optici spricht man beim Vorliegenden folgender Kriterien [4]:

- Alter unter 18 oder über 50 Jahre,

- fehlender Augenbewegungsschmerz,

- simultan beidseitiges Auftreten,

- Papillitis mit starker Schwellung,

Randblutungen, harten Exsudaten,

Cotton-wool-Spots oder initialer

Optikusatrophie.

Erkrankungsmechanismen, die zu einer atypischen Sehnerventzündung führen können und die mittels MRT und Serologie differenziert werden müssen, sind neben der NMOSD Autoimmunerkrankungen (z. B. Sarkoidose, Lupus erythematodes oder „chronic relapsing inflammatory optic neuropathy" [CRION]) sowie ein infektiöses (z.B. Borreliose, Lues oder Bartonellen), postinfektiöses oder postvakzinales Geschehen [4].

Der atypischen NNO im Rahmen von NMOSD gehen häufig grippeähnliche Prodromi voraus. Typischerweise kommt es dann zu einem schnellen beidseitigen Visusverlust, wobei das zweite Auge meist wenige Stunden oder Tage nach dem ersten betroffen ist, was bis zur Erblindung führen kann. Die Papillenschwellung kann mild oder stark ausgeprägt sein. Typisch ist ein schubförmiger Verlauf $(90 \%)$, selten verläuft die Erkrankung monophasisch. Chronisch progrediente Verläufe kommen im Gegensatz zur MS nicht vor [5].

Bei der NMOSD handelt es sich um eine Gruppe seltener chronisch entzündlicher Erkrankungen des zentralen Nervensystems (ZNS). Sie betrifft bevorzugt Frauen (9:1) im mittleren Alter (Altersgipfel 4. Dekade), wobei Erstmanifesta-

Ophthalmologe 2021 · 118:593-596 https://doi.org/10.1007/s00347-020-01165-8

(c) Der/die Autor(en) 2020

R. Yaïci · A. Carzoli · F. Bétis · G. Geerling · R. Guthoff · T. Guthoff

\section{Atypische Neuritis nervi optici (NNO): Bedeutung einer umfassenden Abklärung}

\section{Zusammenfassung}

Eine 65-jährige Frau wurde wegen plötzlicher beidseitiger Sehminderung überwiesen, nachdem sie kurz zuvor geimpft wurde. Augenärztlich zeigte sich beidseits eine ausgeprägte Papillenschwellung. In der Magnetresonanztomografie (MRT) fand sich keine zerebrale Beteiligung oder transverse Myelitis. Serologisch konnten wir Myelin-Oligodendrozyten-Glykoprotein(MOG)-lgG nachweisen, sodass wir mit Hochdosiskortikosteroidpulstherapie behandelten. Diskussion: Bei atypischer Optikusneuritis muss an eine Neuromyelitisoptica-Spektrum-Erkrankung (NMOSD) gedacht werden, die durch die Bestimmung von Aquaporin 4(AQP4)- und MOG-IgG weiter charakterisiert werden sollte.

\section{Schlüsselwörter}

Neuromyelitis optica $\cdot$ NMSOD $\cdot$ Anti-MOGAntikörper · Bilaterales Papillenödem . Postvakzinal

\section{Atypical optic neuritis: the importance of a comprehensive diagnostic work-up}

\begin{abstract}
A 65-year-old woman was referred for sudden bilateral loss of vision. She was vaccinated a few days earlier. The ophthalmological examination showed a massive optic disc swelling on both eyes. Magnetic resonance imaging (MRI) revealed suspected bilateral optic neuritis without cerebral involvement or transverse myelitis. After serological detection of anti-MOG (myelin oligodendrocyte glycoprotein) antibodies, the patient was treated with high-dose corticosteroid pulse
\end{abstract}

therapy until vision recovered. Discussion: an atypical optic neuritis may indicate a neuromyelitis optica spectrum disorder (NMOSD), which should be further characterized by determination of Aquaporin 4(AQP4)-IgG and MOG-lgG.

\section{Keywords}

Neuromyelitis optica $\cdot$ NMSOD $\cdot$ AntiMOG antibodies - Bilateral papilledema . Postvaccination tionen vom Kindes- bis ins hohe Erwachsenenalter beschrieben sind [5]. Neben der NNO können eine Myelitis, ein Areapostrema-Syndrom und die klinische Beteiligung von Hirnstamm, Dienzephalon oder Großhirn einzeln oder in Kombination auftreten [2]. Eine Triggerung der Demyelinisierung durch Impfungen wie bei unserer Patientin ist in einigen Fallberichten beschrieben [3].

Ein wesentliches Diagnosekriterium der NMOSD ist der Serumantikörpernachweis von AQP4-IgG oder MOGIgG, deren Vorhandensein die Erkrankung klar von der MS abgrenzt. AQP4 ist ein Wasserkanalprotein, das beim Menschen in Astrozyten vorkommt [6], es kommt also primär zu einem Astrozytenschaden [2]. AQP4-IgG sind bei NNO als Erstmanifestation einer NMOSD in $70 \%$ nachweisbar. In ca. $25 \%$ der AQP4-nega- tiven Fälle kann man Antikörper gegen MOG nachweisen [5].

MOG ist ein Bestandteil der Myelinscheiden des ZNS. Es spielt wahrscheinlich eine Rolle bei der Adhäsion der Myelinfasern, der Modulation der Interaktion zwischen Myelin und dem Immunsystem und der Stabilität der Oligodendrozyten [2]. Beide Antikörper zusammen kommen nur in Ausnahmefällen beim gleichen Patienten vor.

Das Vorhandensein der unterschiedlichen Antikörper ist klinisch und prognostisch relevant. Es wird kontrovers diskutiert, ob es sich möglicherweise sogar um unterschiedliche Krankheitsentitäten handelt [2]. Die Patienten mit MOG-IgG vermittelter Erkrankung sind meist etwas jünger (Erkrankungsgipfel 3. Dekade), das Geschlechtsverhältnis ist 1:1. Bei ca. $50 \%$ der Patienten ist eine Neuritis 


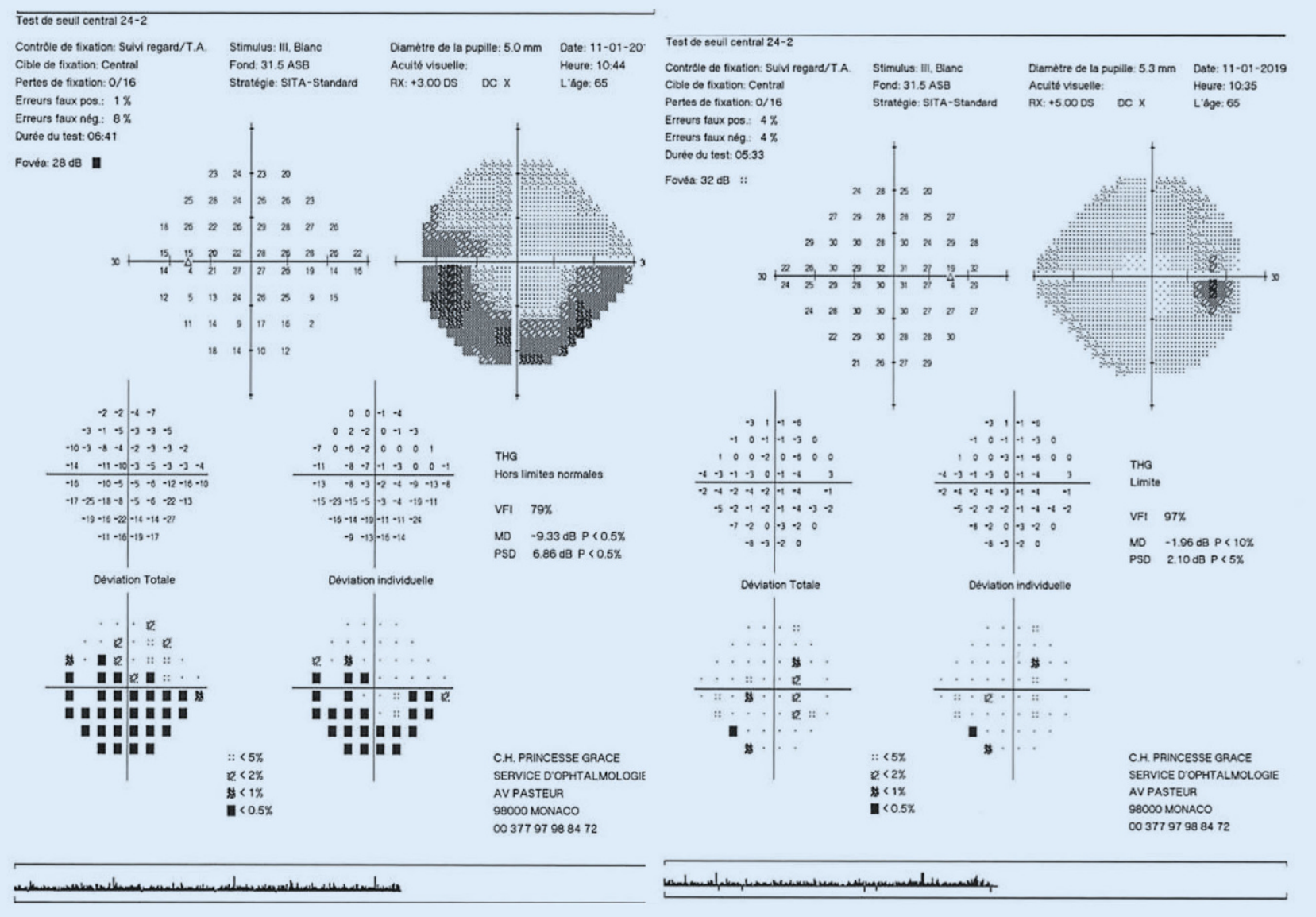

Abb. $2 \Delta$ Statische $30^{\circ}$-Perimetrie 4 Wochen nach Therapie, in welcher sich links ein bogenförmiges Skotom unten mit Beteiligung eines vergrößerten blinden Flecks fand

nervi optici erstes Symptom der Erkrankung und tritt auch im weiteren Verlauf häufig auf. In $50 \%$ ist diese beidseitig und meist mit einer Papillenschwellung verbunden [7].

Während bei AQP4-positiven Patienten die einzelne Neuritisepisode einen stärkeren Sehnervenschaden hinterlässt, scheint es bei MOG-IgG-positiven Patienten zwar zu einer schnelleren Visuserholung, aber zu häufigeren Rezidiven zu kommen, was zu einem vergleichbaren Nervenfaserverlust führt [8].

Die Akuttherapie der NMOSD besteht in Methylprednisolon i.v. $1 \mathrm{~g} / \mathrm{Tag}$ für 3 bis 5 Tage mit mindestens 4-wöchiger Ausschleichphase [2, 9, 10], sofern keine absoluten Kontraindikationen vorliegen. Bei ausbleibender Besserung kann eine Plasmapherese oder Immunadsorption zum Einsatz kommen [2].

Bei AQP4-IgG-positiver NMOSD ist die unvollständige Remission der
Schübe oft Ursache dauerhafter Behinderung, daher ist eine langfristige Immuntherapie zur Schubprävention in den meisten Fällen indiziert. Analog dazu wird auch bei MOG-IgG-positiver NNO mit Azathioprin, Rituximab oder Mycophenolat-Mofetil behandelt, wenn auch eine Langzeittherapie wahrscheinlich nicht bei allen Patienten erforderlich ist und individuell entschieden werden muss. Spezifische Studien, betreffend den Effekt von Rituximab oder neuerer Medikamente wie Interleukin-6-Inhibitoren (Tocilizumab), Komplementsysteminhibitoren (Eculizumab) oder dem seit Juni 2020 von der Federal Drug Administration für AQP4-positive Patienten zugelassenen CD19-Antikörper Inebilizumab-cdon, fehlen bei der Subgruppe der MOG-IgG-positiven Patienten bislang [11].

Einige häufige Immuntherapeutika der MS wie Fingolimod, Interferon- $\beta$ und Natalizumab können eine NMOSD teils massiv verschlechtern [3], auch deshalb ist die Unterscheidung zwischen beiden Erkrankungen wichtig.

Der Augenarzt spielt in der Erkennung der atypischen Neuritis nervi optici eine wichtige Rolle und kann durch eine zielführende Anamnese und Diagnostik die Weichen für die weitere Abklärung stellen.

\section{Fazit für die Praxis}

- Eine Differenzierung zwischen typischer und atypischer Neuritis nervi optici ist für die weitere Therapie essenziell.

- Bei atypischer NNO ist ein wichtiger Teil der Diagnostik die Bestimmung von AQP4-IgG und MOG-IgG.

- Die Evaluation und Therapie der NMOSD muss interdisziplinär erfolgen. 


\section{Korrespondenzadresse}

\section{R. Yaïci}

Klinik für Augenheilkunde, Universitätsklinikum Düsseldorf

Moorenstr. 5, 40225 Düsseldorf, Deutschland remi.yaici@med.uni-duesseldorf.de

Funding. Open Access funding provided by Projekt DEAL.

\section{Einhaltung ethischer Richtlinien}

Interessenkonflikt. R. Yaïci, A. Carzoli, F. Bétis, G. Geerling, R. Guthoff und T. Guthoff geben an, dass kein Interessenkonflikt besteht.

Für diesen Beitrag wurden von den Autoren keine Studien an Menschen oder Tieren durchgeführt. Für die aufgeführten Studien gelten die jeweils dort angegebenen ethischen Richtlinien. Für Bildmaterial oder anderweitige Angaben innerhalb des Manuskripts, über die Patienten zu identifizieren sind, liegt von innen und/oder ihren gesetzlichen Vertretern eine schriftliche Einwilligung vor.

Open Access. Dieser Artikel wird unter der Creative Commons Namensnennung 4.0 International Lizenz veröffentlicht, welche die Nutzung, Vervielfältigung Bearbeitung, Verbreitung und Wiedergabe in jeglichem Medium und Format erlaubt, sofern Sie den/die ursprünglichen Autor(en) und die Quelle ordnungsgemäß nennen, einen Link zur Creative Commons Lizenz beifügen und angeben, ob Änderungen vorgenommen wurden.

Die in diesem Artikel enthaltenen Bilder und sonstige Drittmaterial unterliegen ebenfalls der genannten Creative Commons Lizenz, sofern sich aus der Abbildungslegende nichts anderes ergibt. Sofern das betreffende Material nicht unter der genannten Creative Commons Lizenz steht und die betreffende Handlung nicht nach gesetzlichen Vorschriften erlaubt ist, ist für die oben aufgeführten Weiterverwendungen des Materials die Einwilligung des jeweiligen Rechteinhabers einzuholen.

Weitere Details zur Lizenz entnehmen Sie bitte der Lizenzinformation auf http://creativecommons.org/ licenses/by/4.0/deed.de.

\section{Literatur}

1. Wingerchuk DM et al (2015) International consensus diagnostic criteria for neuromyelitis optica spectrum disorders. Neurology. https://doi. org/10.1212/WNL.0000000000001729

2. dos Passos GR et al (2018) MOG-IgG-associated optic neuritis, encephalitis, and myelitis: lessons learned from neuromyelitis optica spectrum disorder. Front Neurol 9. https://doi.org/10.1186/ s12974-016-0718-0

3. Jarius $S$ et al (2016) MOG-lgG in NMO and related disorders: a multicenter study of 50 patients. Part 2: Epidemiology, clinical presentation, radiological and laboratory features, treatment responses, and long-term outcome. J Neuroinflamm. https://doi org/10.1186/s12974-016-0718-0

4. DOG/BVA Optikusneuritis (2018) Optikusneuritis Synonyme: Neuritis nervi optici, Sehnervenentzündung, Retrobulbärneuritis, englisch: optic neuritis. Leitlinie der DOG/BVA Optikusneuritis, Registernummer 044-010

5. Weinshenker, Wingerchuk (2017) Neuromyelitis spectrum disorders. Mayo Clin Proc 92(4):663-679. https://doi.org/10.1016/j.mayocp.2016.12.014

6. Badaut J, Verbavatz JM, Freund-Mercier MJ, Lasbennes F (2000) Presence of aquaporin-4 and muscarinic receptors in astrocytes and ependymal cells in rat brain: a clue to a common function? Neurosci Lett. https://doi.org/10.1016/S0304 3940(00)01364-1

7. Tajfirouz DA, Bhatti MT, Chen JJ (2019) Clinical characteristics and treatment of MOG-IgG-associated optic neuritis. Curr Neurol Neurosci Rep 19(12). https://doi.org/10.1007/s11910-019-1014-z

8. Pache F et al (2016) MOG-IgG in NMO and related disorders: a multicenter study of 50 patients. Part 4: afferent visual system damage after optic neuritis in MOG-IgG-seropositive versus AQP4IgG-seropositive patients.J Neuroinflamm.https:// doi.org/10.1186/s12974-016-0720-6

9. Jarius S et al (2016) MOG-IgG in NMO and related disorders: a multicenter study of 50 patients. Part 1: Frequency, syndrome specificity, influence of disease activity, long-term course, association with AQP4-IgG, and origin. J Neuroinflamm 13(1):279

10. Azumagawa Ket al (2016) Post-vaccination MDEM associated with MOG antibody in a subclinical chlamydia infected boy. Brain Dev 38(7):690-693

11. Kompetenznetz Multiple Sklerose Qualitätshandbuch MS/NMOSD. www.kompetenznetzmultiplesklerose.de. Zugegriffen: 15. Juni 2020
Franziska-Tiburtius-Preis: Ärztinnen auf neuen Wegen

Ausschreibung

Die Women's Networking Lounge e.V. (WNL) vergibt erstmalig den FranziskaTiburtius-Preis. In Referenz an die Namensgeberin des Preises werden Medizinerinnen gesucht, die neue Wege gehen - Pionierinnen, die etwas wagen. Gefragt sind Präventionskonzepte, Kooperationsmodelle, E-Medizin, soziale Projekte in der Medizin, neue Formen der Patientenkommunikation oder andere innovative Ideen. Teilnehmen können Ärztinnen und Zahnärztinnen aus Deutschland. Der Award ist mit $10.000 €$ dotiert.

Christine Wernze, Vorstandsvorsitzende der WNL zum Award: „Mit der Vergabe dieses Preises geht es uns darum, Ärztinnen zu zeigen, die neben ihrem alltäglich grossartigen Dienst in unserem Gesundheitssystem die Kraft aufbringen, eine besondere Idee zu verfolgen, die das System insgesamt nachhaltig prägt und noch besser macht."

Franziska Tiburtius war die erste Ärztin in Deutschland, als sie sich 1877 in Berlin niederließ. Zuerst führte sie zusammen mit der Zahnärztin Henriette PagelsenHirschfeld eine Gemeinschaftspraxis. Danach eröffnete Franziska Tiburtius mit ihrer Studienkollegin Emilie Lehmus in Prenzlauer Berg eine Poliklinik für Frauen und Kinder. Beide hatten in Zürich studiert, da Frauen bis zu einem entsprechenden Bundesratsbeschluss im Jahr 1899 der Zugang zu deutschen Universitäten verwehrt war.

Bewerbungen

sind bis zum 31.08.2021 über die WNL-Website möglich

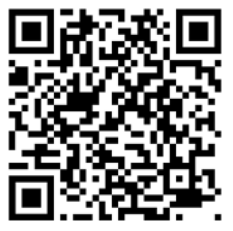

www.womensnetworkinglounge.de 